PROCEEDINGS OF THE

AMERICAN MATHEMATICAL SOCIETY

Volume 135, Number 3, March 2007, Pages 833-836

S 0002-9939(06)08527-3

Article electronically published on September 15, 2006

\title{
THE UNIT BALL OF THE HILBERT SPACE IN ITS WEAK TOPOLOGY
}

\author{
ANTONIO AVILÉS
}

(Communicated by N. Tomczak-Jaegermann)

\begin{abstract}
We show that the unit ball of $\ell_{p}(\Gamma)$ in its weak topology is a continuous image of $\sigma_{1}(\Gamma)^{\mathbb{N}}$, and we deduce some combinatorial properties of its lattice of open sets which are not shared by the balls of other equivalent norms when $\Gamma$ is uncountable.
\end{abstract}

For a set $\Gamma$ and a real number $1<p<\infty$, the Banach space $\ell_{p}(\Gamma)$ is a reflexive space, hence its unit ball is compact in the weak topology, and in fact, it is homeomorphic to the following closed subset of the Tychonoff cube $[-1,1]^{\Gamma}$ :

$$
B(\Gamma)=\left\{x \in[-1,1]^{\Gamma}: \sum_{\gamma \in \Gamma}\left|x_{\gamma}\right| \leq 1\right\} .
$$

The homeomorphism $h: B_{\ell_{p}(\Gamma)} \longrightarrow B(\Gamma)$ is given by $h(x)_{\gamma}=\operatorname{sign}\left(x_{\gamma}\right) \cdot\left|x_{\gamma}\right|^{p}$. The spaces homeomorphic to closed subsets of some $B(\Gamma)$ constitute the class of uniform Eberlein compacta, introduced by Benyamini and Starbird 6]. The space $\sigma_{k}(\Gamma)$, the compact subset of $\{0,1\}^{\Gamma}$ which consists of the functions with at most $k$ nonzero coordinates ( $k$ a positive integer), is an example of a uniform Eberlein compact. In fact, the following result was proven in [5].

Theorem 1 (Benyamini, Rudin, Wage). Every uniform Eberlein compact of weight $\kappa$ is a continuous image of a closed subset of $\sigma_{1}(\kappa)^{\mathbb{N}}$.

In the same paper [5], the problem was posed of whether it was possible to get any uniform Eberlein compact as a continuous image of the full $\sigma_{1}(\Gamma)^{\mathbb{N}}$. This question was answered in the negative by Bell [2, by considering the following property:

A compact space $K$ verifies property (Q) if for every uncountable regular cardinal $\lambda$ and every family $\left\{U_{\alpha}, V_{\alpha}\right\}_{\alpha<\lambda}$ of open subsets of $K$ with $\overline{U_{\alpha}} \subset V_{\alpha}$ one of the following two alternatives must hold:

(1) either there exists a set $A \subset \lambda$ with $|A|=\lambda$ such that $U_{\alpha} \cap U_{\beta}=\emptyset$ for every two different elements $\alpha$ and $\beta$ in $A$,

(2) or there exists a set $A \subset \lambda$ with $|A|=\lambda$ such that $V_{\alpha} \cap V_{\beta} \neq \emptyset$ for every two different elements $\alpha$ and $\beta$ in $A$.

Received by the editors March 2, 2005 and, in revised form, October 19, 2005.

2000 Mathematics Subject Classification. Primary 46B50, 46B26, 46C05, 54B30, 54D15.

Key words and phrases. Uniform Eberlein compact, polyadic space, $\ell_{p}$ spaces, Hilbert space, weak topology, equivalent norm.

The author was supported by an FPU grant of MEC of Spain.

(C)2006 American Mathematical Society 
Bell proved in 2 that property (Q) is satisfied by all polyadic spaces, that is, continuous images of $\sigma_{1}(\Gamma)^{\Lambda}$ for any sets $\Gamma$ and $\Lambda$ (this concept was introduced in [8] and studied earlier by Gerlits [7]), but he constructed a uniform Eberlein compact without property (Q). Later, Bell 4 provided another example of a uniform Eberlein compact which is not a continuous image of any $\sigma_{1}(\Gamma)^{\mathbb{N}}$ but which is nevertheless polyadic. Our main result is the following.

Theorem 2. $B(\Gamma)$ is a continuous image of $\sigma_{1}(\Gamma)^{\mathbb{N}}$.

As a consequence, $B(\Gamma)$ satisfies property $(\mathrm{Q})$ as well as other properties of the same type introduced by Bell in $[4$ and 3 . However, if $\Gamma$ is uncountable, we show in Theorem 4 that a modification of one of the examples of Bell provides an equivalent norm on $\ell_{p}(\Gamma)$ whose unit ball is not a continuous image of $\sigma_{1}(\Gamma)^{\mathbb{N}}$, indeed not satisfying property $(\mathrm{Q})$. In particular, we are showing the existence of equivalent norms in the nonseparable $\ell_{p}(\Gamma)$ whose closed unit balls are not homeomorphic in the weak topology. This contrasts with the separable case, since the balls of all separable reflexive Banach spaces are weakly homeomorphic [1, Theorem 1.1]. We refer to [1] for information about the problem of whether the balls of equivalent norms in a Banach space are weakly homeomorphic in the separable case.

Proof of Theorem 2. For a set $\Delta$, we will use the notation $B^{+}(\Delta)=B(\Delta) \cap[0,1]^{\Delta}$. First, we point out that $B(\Gamma)$ is a continuous image of $B^{+}(\Gamma)$. Indeed, if we consider $\Gamma^{o}=\Gamma \times\{a, b\}$, we have a continuous surjection $\psi: B^{+}\left(\Gamma^{o}\right) \longrightarrow B(\Gamma)$ given by $\psi(x)_{\gamma}=x_{(\gamma, a)}-x_{(\gamma, b)}$.

In a second step, we apply the standard procedure to express the space $B^{+}(\Gamma)$ as a continuous image of a totally disconnected compact $L_{0}$. We fix a sequence $\left(r_{n}\right)_{n=0}^{\infty}$ of positive real numbers such that $\sum_{n=0}^{\infty} r_{n}=1$ and such that the continuous map $\phi:\{0,1\}^{\mathbb{N}} \longrightarrow[0,1]$ given by $\phi(x)=\sum_{n=0}^{\infty} r_{n} x_{n}$ is surjective, for example, $r_{n}=\frac{1}{2^{n+1}}$. We consider the power $\phi^{\Gamma}:\{0,1\}^{\Gamma \times \mathbb{N}} \longrightarrow[0,1]^{\Gamma}$ and then we set:

$$
\begin{aligned}
L_{0} & =\left(\phi^{\Gamma}\right)^{-1}\left(B^{+}(\Gamma)\right), \\
f & =\left.\phi^{\Gamma}\right|_{L_{0}},
\end{aligned}
$$

so that $f: L_{0} \longrightarrow B^{+}(\Gamma)$ is a continuous surjection. It will be convenient to have an explicit description of $L_{0}$. For $x \in\{0,1\}^{\Gamma \times \mathbb{N}}$ and $n \in \mathbb{N}$, we define $N_{n}(x)=$ $\left|\left\{\gamma \in \Gamma: x_{(\gamma, n)}=1\right\}\right|$.

$$
\begin{aligned}
x \in L_{0} & \Longleftrightarrow \phi^{\Gamma}(x) \in B^{+}(\Gamma) \\
& \Longleftrightarrow \sum_{\gamma \in \Gamma} \phi^{\Gamma}(x)_{\gamma} \leq 1 \\
& \Longleftrightarrow \sum_{\gamma \in \Gamma} \sum_{n=0}^{\infty} r_{n} x_{(\gamma, n)} \leq 1 \\
& \Longleftrightarrow \sum_{n=0}^{\infty} r_{n} N_{n}(x) \leq 1 .
\end{aligned}
$$

The compact space $L_{0}$ can be alternatively described as follows. Let $Z$ be a compact subset of $\mathbb{N}^{\mathbb{N}}$ such that if $\sigma \in Z$ and $\tau_{n} \leq \sigma_{n}$ for all $n \in \mathbb{N}$, then $\tau \in Z$. Associated to such a set $Z$ we construct the following space:

$$
\mathcal{K}(Z, \Gamma)=\left\{x \in\{0,1\}^{\Gamma \times \mathbb{N}}:\left(N_{n}(x)\right)_{n \in \mathbb{N}} \in Z\right\} .
$$


We have that $L_{0}=\mathcal{K}\left(Z_{0}, \Gamma\right)$ where $Z_{0}=\left\{s \in \mathbb{N}^{\mathbb{N}}: \sum_{i \in \mathbb{N}} r_{i} s_{i} \leq 1\right\}$. Note that $Z_{0}$ is indeed compact since it is a closed subset of $\prod_{n \in \mathbb{N}}\left\{0, \ldots, M_{n}\right\}$ where $M_{n}$ is the integer part of $\frac{1}{r_{n}}$. The proof will be complete after the following lemma.

Lemma 3. Let $Z$ be a compact subset of $\mathbb{N}^{\mathbb{N}}$ such that if $\sigma \in Z$ and $\tau_{n} \leq \sigma_{n}$ for all $n \in \mathbb{N}$, then $\tau \in Z$. Then $\mathcal{K}(Z, \Gamma)$ is a continuous image of $\sigma_{1}(\Gamma)^{\mathbb{N}}$.

Proof. First we check that $\mathcal{K}(Z, \Gamma)$ is a closed subset of $\{0,1\}^{\Gamma \times \mathbb{N}}$ and hence compact. Namely, if $x \in\{0,1\}^{\Gamma \times \mathbb{N}} \backslash \mathcal{K}(Z, \Gamma)$, then $\left(N_{n}(x)\right)_{n \in \mathbb{N}} \notin Z$ and since $Z$ is closed in $\mathbb{N}^{\mathbb{N}}$, there is a finite set $F \subset \mathbb{N}$ such that $\sigma \notin Z$ whenever $\sigma_{n}=N_{n}(x)$ for all $n \in F$. Indeed, by the assumptions on $Z$, if $\tau \in \mathbb{N}^{\mathbb{N}}$ and $\tau_{n} \geq \sigma_{n}$ for all $n \in F$, then also $\tau \notin Z$. In this case,

$$
W=\left\{y \in\{0,1\}^{\Gamma \times \mathbb{N}}: y_{\gamma, n}=1 \text { whenever } n \in F \text { and } x_{\gamma, n}=1\right\}
$$

is a neighborhood which separates $x$ from $\mathcal{K}(Z, \Gamma)$ and this finishes the proof that $\mathcal{K}(Z, \Gamma)$ is closed. Since $Z$ is compact, for every $n \in \mathbb{N}$ there exists $M_{n} \in \mathbb{N}$ such that $\sigma_{n} \leq M_{n}$ for all $\sigma \in Z$. We define the following compact space:

$$
L_{1}=Z \times \prod_{m \in \mathbb{N}} \prod_{i=0}^{M_{m}} \sigma_{i}(\Gamma) .
$$

Note that $L_{1}$ is a continuous image of $\sigma_{1}(\Gamma)^{\mathbb{N}}$. On the one hand, since $Z$ is a metrizable compact, it is a continuous image of $\{0,1\}^{\mathbb{N}}$ and in particular of $\sigma_{1}(\Gamma)^{\mathbb{N}}$. On the other hand, for any $i \in \mathbb{N}$, the space $\sigma_{i}(\Gamma)$ can be viewed as the family of all subsets of $\Gamma$ of cardinality at most $i$. In this way, we consider the continuous surjection $p: \sigma_{1}(\Gamma)^{i} \longrightarrow \sigma_{i}(\Gamma)$ given by $p\left(x_{1}, \ldots, x_{i}\right)=x_{1} \cup \cdots \cup x_{i}$. From the existence of such a function follows the fact that any countable product of spaces $\sigma_{i}(\Gamma)$ is a continuous image of $\sigma_{1}(\Gamma)^{\mathbb{N}}$, and in particular, the second factor in the expression of $L_{1}$ is such an image.

It remains to define a continuous surjection $g: L_{1} \longrightarrow \mathcal{K}(Z, \Gamma)$. We first fix some notation. An element of $L_{1}$ will be written as $(z, x)$ where $z \in Z$ and $x \in$ $\prod_{m \in \mathbb{N}} \prod_{i=0}^{M_{m}} \sigma_{i}(\Gamma)$. At the same time, such an $x$ is of the form $\left(x^{m}\right)_{m \in \mathbb{N}}$ with $x^{m} \in \prod_{i=0}^{M_{m}} \sigma_{i}(\Gamma)$ and again each $x^{m}$ is $\left(x^{m, i}\right)_{i=1}^{M_{m}}$ where $x^{m, i} \in \sigma_{i}(\Gamma)$. Finally $x^{m, i}=\left(x_{\gamma}^{m, i}\right)_{\gamma \in \Gamma} \in \sigma_{i}(\Gamma) \subset\{0,1\}^{\Gamma}$. The function $g: L_{1} \longrightarrow \mathcal{K}(Z, \Gamma) \subset\{0,1\}^{\Gamma \times \mathbb{N}}$ is defined as follows:

$$
g(z, x)_{\gamma, m}=x_{\gamma}^{m, z(m)} .
$$

Observe that $g(x, z)$ maps $L_{1}$ onto $\mathcal{K}(Z, \Gamma)$ because for every $m,\left(x_{\gamma}^{m, z(m)}\right)_{\gamma \in \Gamma}$ is an arbitrary element of $\sigma_{z(m)}(\Gamma)$.

Theorem 4. Let $\Gamma$ be an uncountable set and $1<p<\infty$. There exists an equivalent norm on $\ell_{p}(\Gamma)$ whose unit ball does not satisfy property $(Q)$ and hence it is not polyadic.

Proof. This is a variation of an example of Bell [2], originally a scattered compact, that makes it absolutely convex. We will consider $\omega_{1}$ as a subset of $\Gamma$. Let $\phi$ : $\omega_{1} \longrightarrow \mathbb{R}$ be a one-to-one map and

$$
G=\left\{(\alpha, \beta) \in \omega_{1} \times \omega_{1}: \phi(\alpha)<\phi(\beta) \Longleftrightarrow \alpha \preceq \beta\right\} .
$$

We define an equivalent norm on $\ell_{p}(\Gamma) \times \ell_{p}(\Gamma) \sim \ell_{p}(\Gamma)$ by

$$
\|(x, y)\|^{\prime}=\sup \left\{\|x\|_{p},\|y\|_{p},\left|x_{\alpha}\right|+\left|y_{\beta}\right|:(\alpha, \beta) \in G\right\},
$$


and let $K$ be its unit ball considered in its weak topology. Fix numbers $1<\xi_{1}<$ $\xi_{2}<2^{1-\frac{1}{p}}$. The families of open sets

$$
\begin{aligned}
& U_{\alpha}=\left\{(x, y) \in K:\left|x_{\alpha}\right|+\left|y_{\alpha}\right|>\xi_{2}\right\}, \quad \alpha<\omega_{1}, \\
& V_{\alpha}=\left\{(x, y) \in K:\left|x_{\alpha}\right|+\left|y_{\alpha}\right|>\xi_{1}\right\}, \quad \alpha<\omega_{1} \text {, }
\end{aligned}
$$

verify that $\overline{U_{\alpha}} \subset V_{\alpha}$ and that for any $\alpha, \beta<\omega_{1}, U_{\alpha} \cap U_{\beta}=\emptyset$ if and only if $(\alpha, \beta) \in G$ if and only if $V_{\alpha} \cap V_{\beta}=\emptyset$. Namely, if there is some $(x, y) \in V_{\alpha} \cap V_{\beta}$, then

$$
\left|x_{\alpha}\right|+\left|y_{\alpha}\right|+\left|x_{\beta}\right|+\left|y_{\beta}\right|>\xi_{1}+\xi_{1}>2
$$

and therefore either $\left|x_{\alpha}\right|+\left|x_{\beta}\right|>1$ or $\left|y_{\alpha}\right|+\left|y_{\beta}\right|>1$ and this implies that $(\alpha, \beta) \notin G$ since $(x, y) \in K$. On the other hand, if $(\alpha, \beta) \notin G$, then the element $(x, y) \in$ $\ell_{p}(\Gamma) \times \ell_{p}(\Gamma)$ which has all coordinates zero except $x_{\alpha}=x_{\beta}=y_{\alpha}=y_{\beta}=2^{-\frac{1}{p}}$ lies in $U_{\alpha} \cap U_{\beta}$. Since there is no uncountable well-ordered (or inversely well-ordered) subset of $\mathbb{R}$, there is no uncountable subset $A$ of $\omega_{1}$ such that $A \times A \subset G$ or $(A \times A) \cap G=\emptyset$. Therefore, the families $\left\{U_{\alpha}\right\}$ and $\left\{V_{\alpha}\right\}$ witness the fact that $K$ does not have property $(\mathrm{Q})$ and hence, it is not polyadic.

The present work was written during a visit to the University of Warsaw, which the author thanks for its hospitality. In particular, the author thanks Witold Marciszewski and Roman Pol from the University of Warsaw, and Rafał Górak from the Polish Academy of Sciences. This work owes very much to a discussion with them and their suggestions.

\section{REFERENCES}

1. T. Banakh, The topological classification of weak unit balls of Banach spaces, Dissertationes Math. (Rozprawy Mat.) 387 (2000), 7-35. MR.1759047 (2001f:57022)

2. M. Bell, A Ramsey theorem for polyadic spaces, Fund. Math. 150 (1996), no. 2, 189-195. MR.1391299 (97d:54042)

3. - On character and chain conditions in images of products, Fund. Math. 158 (1998), no. 1, 41-49. MR 1641145 (99h:54036)

4. _ Polyadic spaces of countable tightness, Topology Appl. 123 (2002), no. 3, 401-407. MR:1924040 (2004f:54005)

5. Y. Benyamini, M. E. Rudin, and M. Wage, Continuous images of weakly compact subsets of Banach spaces, Pacific J. Math. 70 (1977), no. 2, 309-324. MR0625889 (58:30065)

6. Y. Benyamini and T. Starbird, Embedding weakly compact sets into Hilbert space, Israel J. Math. 23 (1976), no. 2, 137-141. MR0397372 (53:1231)

7. J. Gerlits, On a generalization of dyadicity, Studia Sci. Math. Hungar. 13 (1978), no. 1-2, 1-17 (1981). MR0630375 (83e:54019)

8. S. Mrówka, Mazur theorem and m-adic spaces, Bull. Acad. Polon. Sci. Sér. Sci. Math. Astronom. Phys. 18 (1970), 299-305. MR0264613(41:9205)

Departamento de Matemáticas, Universidad de Murcia, 30100 Espinardo (Murcia), SPAIN

E-mail address: avileslo@um.es

Current address: Equipe de Logique Mathematique, Université de Paris 7, Place Jussieu, 2, 75251 Paris, France 